\title{
A Control Volume Based Finite Element Method for Plane Micropolar Elasticity
}

\author{
M.A. Wheel \\ Department of Mechanical Engineering, \\ University of Strathclyde, \\ Montrose Street, Glasgow, G1 1XJ, UK \\ marcus.wheel@strath.ac.uk, Tel:- +44 141548 3307, Fax:- +44 1415525105
}

\section{Summary}

This paper describes the development of a numerical procedure for predicting deformations and stresses in a loaded two dimensional membrane exhibiting micropolar or Cosserat constitutive behaviour. The procedure employs a conventional finite element mesh together with a dual mesh of interconnected control volumes, each of which must satisfy equilibrium. A series of patch tests covering a variety of simple strain states are used to validate the procedure which is then employed to predict the stress concentration in a membrane containing a small hole. The predictions provided by the procedure are compared with those given previously by finite elements.

Keywords: micropolar, Cosserat, finite element, control volume

\section{Introduction}

Classical elasticity theory is regarded as local in the sense that the state of stress at a point in a loaded material is related to the strain at that point through a suitable constitutive relationship. Its popularity persists because it provides a perfectly adequate description of the response of many materials when loaded. This can be attributed to the fact that although these materials are actually heterogeneous when viewed at the microscopic level they appear homogeneous at the larger scales that are usually of interest. However, there are instances where the assumption of material homogeneity is inadequate: either the size of the loaded structure is very small and comparable to the length scale of its constituent material microstructure or the length scale of the heterogeneity with the material structure is significantly larger than microscopic. Many nanotechnological devices fall into the first category while materials such as ceramics, cement, rock, soil, bone and short fibre and particulate reinforced composites are members of the second category.

Alternative theories describing the behaviour of heterogeneous materials are available. These theories are regarded as nonlocal because they assume that the state of stress at a point not only depends on the strain at that point but also on the strain in the surrounding neighbourhood. Nonlocal theories therefore incorporate the concept of a length scale that quantifies the size of this neighbourhood and thereby recognises the underlying structure of the material. Gradient theories incorporate nonlocality by relating the stress to not only first but also higher order derivatives of displacement [1]. Micropolar or Cosserat theories introduce couple stresses that supplement the conventional stresses and are related to additional internal rotational degrees of freedom through constitutive relations that include a length scale parameter [2]. 
Incorporating nonlocal theories into numerical methods for predicting the behaviour of loaded heterogeneous materials is not as straightforward as with classical elasticity theory. Gradient theories ideally require inter element continuity of higher order displacement derivatives and this is not generally exhibited by conventional finite element (FE) methods. The development of suitable elements offering this feature is actively being pursued [3, 4, 5]. Micropolar elasticity theory has been incorporated into FE methods by a number of researchers $[6,7,8,9,10]$. However, the numerical results reported indicate that there are some issues that arise. Firstly, the accuracy of the numerical solutions can vary as the values of the additional micropolar elasticity parameters are changed even though the mesh remains unaltered $[7,8,10]$. Secondly, some elements appear to only satisfy appropriate patch tests approximately rather than exactly as might be expected [8]. Finally, reported results invariably consider two dimensional cases only implying that full three dimensional analyses are an as yet unrealized challenge for FE methods.

This paper describes the initial development of an alternative numerical approach to predicting deformations in loaded heterogeneous materials that exhibit micropolar elastic behaviour. The description given here concentrates on the case of planar deformations; the extension of the approach to the more general three dimensional situation forms the basis of ongoing research. The approach itself employs a conventional finite element mesh with the unknown degrees of freedom located at the element vertices. However, instead of deriving the algebraic equations for the vertex unknowns by minimizing total potential energy they are obtained by imposing equilibrium locally on control volumes constructed around each element vertex. This so called control volume based finite element method (CVFEM) was first developed for convection diffusion problems [11] because its geometric versatility provided the opportunity to represent complicated flow domains while preserving the local conservation property of earlier Cartesian grid based finite volume methods [12]. The CVFEM has also been referred to as the vertex centred edge based finite volume method [13]. Lately the development of computational methods for analysing phenomena like melting and solidification or flow induced vibration, where different physical media are interacting, has produced control volume based procedures for numerical solid mechanics [14] that are compatible with their computational fluid dynamics (CFD) counterparts. These procedures have been used to analyse various fluid structure interaction problems $[15,16,17]$. Control volume procedures have also been employed in the solid mechanics context to analyse specific problems such as fracture [18], where the intrinsic local conservation feature can usefully be exploited in the crack tip neighbourhood, and in analysing nonlinear problems [19,20,21], where CFD experience, particularly in solving the discrete equations, can be reutilized. In general, the procedures usually incorporate low order displacement variations and provide displacement, strain and stress field predictions that are of similar accuracy to those given by corresponding FE methods. However, a recently developed CVFEM procedure incorporating additional rotational degrees of freedom and associated quadratic displacement variations [22] produced displacement predictions that were noticeably more accurate than its FE equivalent [23]. The research described here also includes vertex rotations but rather than simply providing higher order variations in displacement these now represent the microrotations intrinsically present in micropolar elasticity theory. This paper first provides a brief summary of plane micropolar or Cosserat elasticity theory and then describes the 
formulation of the CVFEM in some detail. Finally, numerical results for a test problem are presented and compared with available FE results.

\section{Overview of Plane Micropolar Elasticity}

Micropolar or Cossarat elasticity extends classical elasticity theory by incorporating additional couples per unit area that act on a material element. These couple stresses, $m_{x}$ and $m_{y}$, together with the conventional direct stress components, $\sigma_{x}$ and $\sigma_{y}$, and shear stress components, $\tau_{x y}$ and $\tau_{y x}$, are shown acting on a loaded two dimensional element in Figure 1.

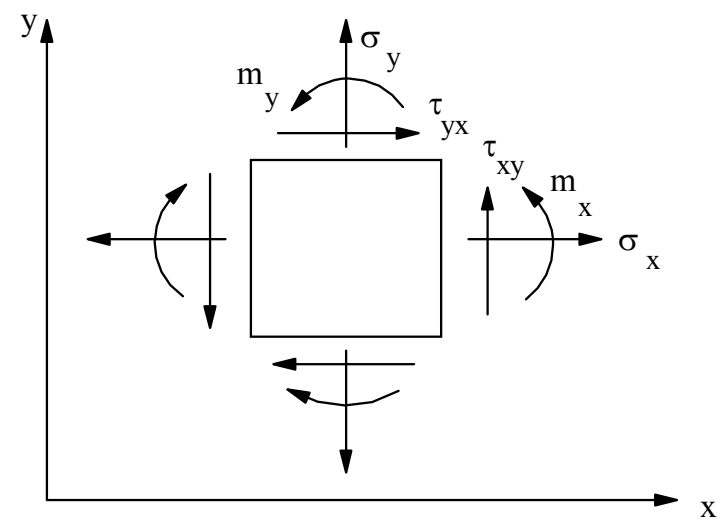

Figure 1 Stress system for a micropolar material element

Equilibrium of the element in the Cartesian coordinate directions yields the following pair of equilibrium equations:-

$$
\begin{aligned}
& \sigma_{x, x}+\tau_{y x, y}+p_{x}=0 \\
& \tau_{x y, x}+\sigma_{y, y}+p_{y}=0
\end{aligned}
$$

where $p_{x}$ and $p_{y}$ are the components of any body force per unit volume that may be imposed on the element and the comma in the subscript denotes differentiation with respect to the succeeding variable in the usual way. Equilibrium of moments about the centre of the element yields a third equilibrium equation:-

$$
m_{x, x}+m_{y, y}+\tau_{x y}-\tau_{y x}+q=0
$$

where $q$ represents a body moment per unit volume that may be acting on the element. Equation 3 implies that when the couple stresses are present the shear stresses do not need to complement one another and are therefore not necessarily equal. The shear stresses can be written in terms of a symmetric component, $\tau_{s}$, and an antisymmetric component, $\tau_{a}:-$

where

$$
\begin{aligned}
& \tau_{x y}=\tau_{s}+\tau_{a} \\
& \tau_{y x}=\tau_{s}-\tau_{a} \\
& \tau_{s}=1 / 2\left(\tau_{x y}+\tau_{y x}\right)
\end{aligned}
$$


and

$$
\tau_{a}=1 / 2\left(\tau_{x y}-\tau_{y x}\right)
$$

The symmetric component is related to conventional shear deformation in the element while the antisymmetric component is associated with a rotation, $\phi$, of the element as shown in figure 2. In Cosserat elasticity this microrotation is independent of displacements; it is not equal to the conventional rotation represented by the curl of the displacement vector. Constraining the microrotation to equal the conventional rotation leads to a simplified couple stress theory of elasticity.

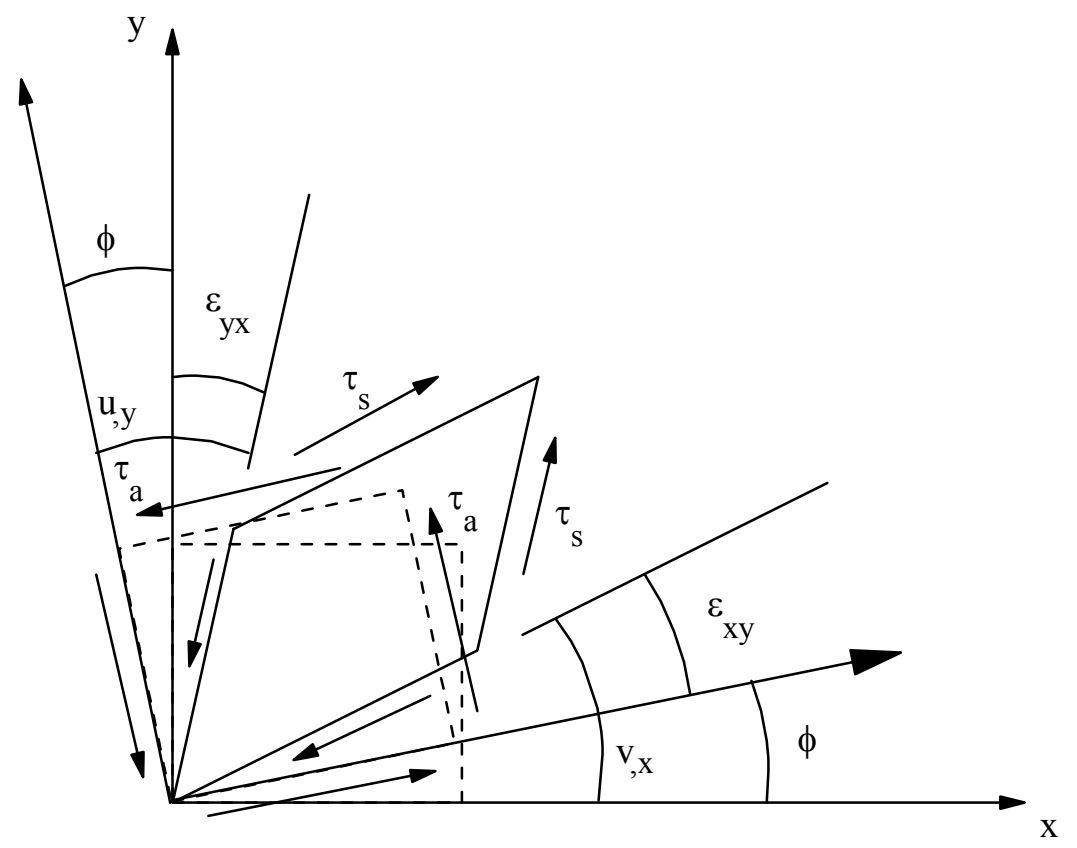

Figure 2 Deformations associated with shear stress components

In order to solve the equilibrium equations 1 to 3 numerically using a CVFEM employing displacement and rotation variables it is necessary to link the applied stresses to the resulting deformations via a set of constitutive relationships. In the plane stress case these are [24]:-

and

$$
\begin{gathered}
\left(\begin{array}{l}
\sigma_{x} \\
\sigma_{y} \\
\tau_{y x} \\
\tau_{x y}
\end{array}\right)=\frac{E_{m}}{\left(1-v_{m}^{2}\right)}\left(\begin{array}{cccc}
1 & v_{m} & 0 & 0 \\
v_{m} & 1 & 0 & 0 \\
0 & 0 & \frac{\left(1-v_{m}\right)}{2\left(1-N^{2}\right)} & \frac{\left(1-v_{m}\right)\left(1-2 N^{2}\right)}{2\left(1-N^{2}\right)} \\
0 & 0 & \frac{\left(1-v_{m}\right)\left(1-2 N^{2}\right)}{2\left(1-N^{2}\right)} & \frac{\left(1-v_{m}\right)}{2\left(1-N^{2}\right)}
\end{array}\right)\left(\begin{array}{c}
\varepsilon_{x} \\
\varepsilon_{y} \\
\varepsilon_{y x} \\
\varepsilon_{x y}
\end{array}\right) \\
\left(\begin{array}{l}
m_{x} \\
m_{y}
\end{array}\right)=\left(\begin{array}{cc}
\frac{2 E_{m} l^{2}}{1+v_{m}} & 0 \\
0 & \frac{2 E_{m} l^{2}}{1+v_{m}}
\end{array}\right)\left(\begin{array}{l}
\phi_{, x} \\
\phi_{, y}
\end{array}\right)
\end{gathered}
$$

In the plane strain case the direct and shear stress components are given by [24] :- 


$$
\left(\begin{array}{c}
\sigma_{x} \\
\sigma_{y} \\
\tau_{y x} \\
\tau_{x y}
\end{array}\right)=\frac{E_{m}\left(1-v_{m}\right)}{\left(1+v_{m}\right)\left(1-2 v_{m}\right)}\left(\begin{array}{cccc}
1 & \frac{v_{m}}{1-v_{m}} & 0 & 0 \\
\frac{v_{m}}{1-v_{m}} & 1 & 0 & 0 \\
0 & 0 & \frac{1-2 v_{m}}{2\left(1-v_{m}\right)\left(1-N^{2}\right)} & \frac{\left(1-2 v_{m}\right)\left(1-2 N^{2}\right)}{2\left(1-v_{m}\right)\left(1-N^{2}\right)} \\
0 & 0 & \frac{\left(1-2 v_{m}\right)\left(1-2 N^{2}\right)}{2\left(1-v_{m}\right)\left(1-N^{2}\right)} & \frac{1-2 v_{m}}{2\left(1-v_{m}\right)\left(1-N^{2}\right)}
\end{array}\right)\left(\begin{array}{c}
\varepsilon_{x} \\
\varepsilon_{y} \\
\varepsilon_{y x} \\
\varepsilon_{x y}
\end{array}\right)
$$

while the couple stresses are still given by equation 9 . Here $\varepsilon_{x}$ and $\varepsilon_{y}$ are the direct strain components and $\varepsilon_{x y}$ and $\varepsilon_{y x}$ are the shear strains. The rotation derivatives appearing in equation 9 are referred to as the curvatures as in plate theory. The subscript $m$ is added to $E$, the Young's modulus, and $v$, the Poisson's ratio to acknowledge that they are the micropolar material properties that would be obtained from a uniaxial load test in which a constant state of strain is induced throughout the test piece. The other two material properties appearing in these relationships are the characteristic length, $l$, and the parameter $N$, termed the 'coupling factor', which quantifies the interaction intensity between the displacement and rotation fields [24]. An alternative interpretation of this interaction can be obtained by letting:-

$$
N^{2}=\frac{a}{1+a}
$$

and, by noting that a shear modulus, $G_{m}$ can be defined in terms of $E_{m}$ and $v_{m}$ thus:-

$$
G_{m}=\frac{E_{m}}{2\left(1+v_{m}\right)},
$$

then the last two relationships in equation 10 can be simplified to:-

$$
\left(\begin{array}{c}
\tau_{y x} \\
\tau_{x y}
\end{array}\right)=G_{m}\left(\begin{array}{ll}
1+a & 1-a \\
1-a & 1+a
\end{array}\right)\left(\begin{array}{l}
\varepsilon_{y x} \\
\varepsilon_{x y}
\end{array}\right)
$$

as in [8]. The strains appearing in equation 10 are related to the displacements by:-

$$
\left(\begin{array}{c}
\varepsilon_{x} \\
\varepsilon_{y} \\
\varepsilon_{y x} \\
\varepsilon_{x y}
\end{array}\right)=\left(\begin{array}{c}
u_{, x} \\
v_{, y} \\
u_{, y}+\phi \\
v_{, x}-\phi
\end{array}\right)
$$

where $u$ and $v$ are the displacement components in the $x$ and $y$ directions respectively. By substituting the expressions for the shear strains given in 14 into 13 it is possible to show using 6 and 7 that the antisymmetric component of the shear stress depends 
on $\phi$ and $a$ (and thereby $N$ ) while the symmetric component is independent of them. Thus $a$ may be regarded as a parameter that characterises the relative magnitudes of the symmetric and antisymmetric components of the shear stress. The lower bound on $a$ occurs when $a=0$ (or $N=0$ ) and corresponds to classical elasticity while the upper bound, $a=\infty$ (or $N=1$ ), is equivalent to couple stress theory.

\subsection{The CVFEM Formulation}

\subsection{The control volume dual mesh}

The geometry of a two dimensional structure is represented by a mesh of finite elements that are connected to one another at their vertices in the usual manner. The shape of the elements is, in general, not restricted; they can be triangular, quadrilateral or even polygonal. A dual mesh of control volumes (CVs) is constructed by connecting each element centre to the midpoints of its edges. Figure 3 shows the case where a typical $\mathrm{CV}$ belonging to the dual mesh is formed around one of the vertices located in a mesh of triangular elements. In general, $\mathrm{n}$ elements will be connected to the vertex so the $\mathrm{CV}$ forms a multifaceted polygon with $2 \mathrm{n}$ edges numbered from 1 to $2 \mathrm{n}$ as illustrated. It is important to note that the dual mesh is conceptual; in practice it is unnecessary to store any information about its construction as will be explained later.

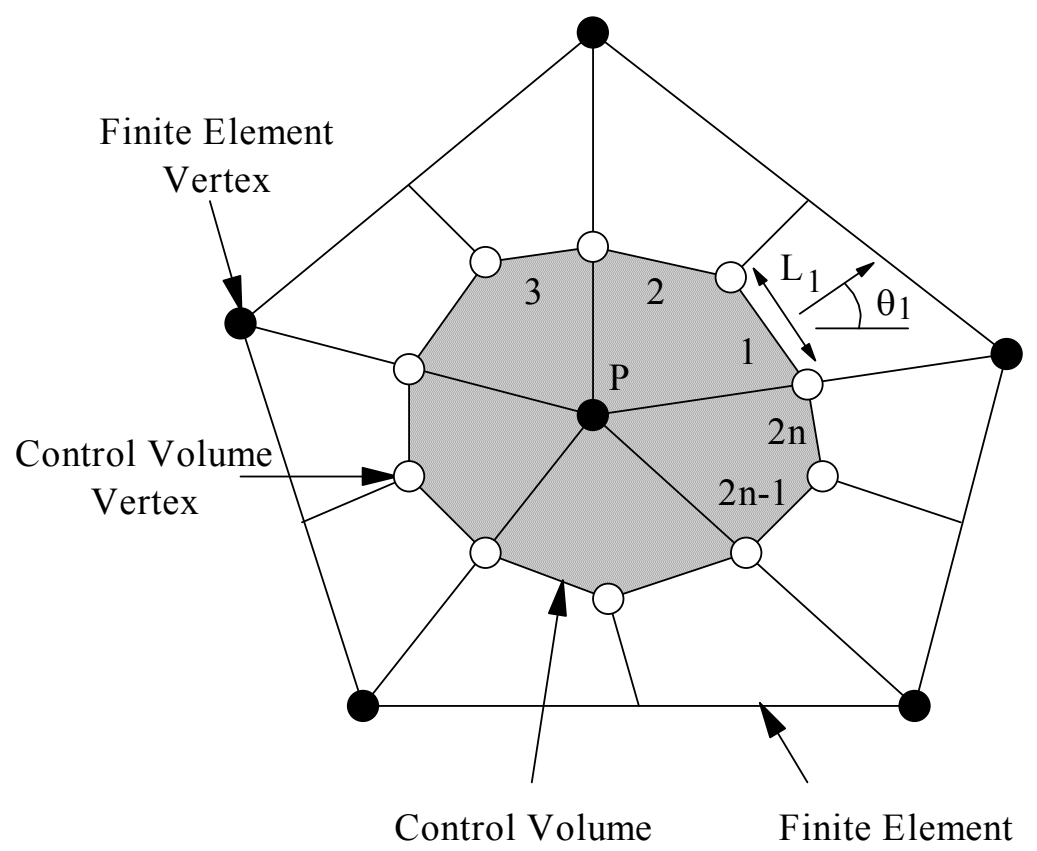

Fig.3 Multifaceted control volume constructed around element vertex

Equilibrium equations 1, 2 and 3 are satisfied by each $\mathrm{CV}$ when the resultants of the stresses acting on their boundaries balance any body loadings imposed on them. Equilibrium of a particular CV can thus be represented by:-

$$
\sum_{j=1}^{j=2 n} F_{x}^{j}+p_{x} A_{V}=0
$$




$$
\sum_{j=1}^{j=2 n} F_{y}^{j}+p_{y} A_{V}=0
$$

and

$$
\sum_{j=1}^{j=2 n} M_{P}^{j}+q A_{V}=0
$$

where $F_{x}^{j}$ and $F_{y}^{j}$ are the components of the stress resultants acting on face $\mathrm{j}$ of the volume, $M_{P}^{j}$ is the resultant moment about $\mathrm{P}$ of the stresses and $A_{V}$ is the area of the CV.

\subsection{The triangular finite elements}

A typical triangular finite element with three degrees of freedom per vertex, two displacement components, $u$ and $v$, and a rotation $\phi$, is shown in Figure 4.

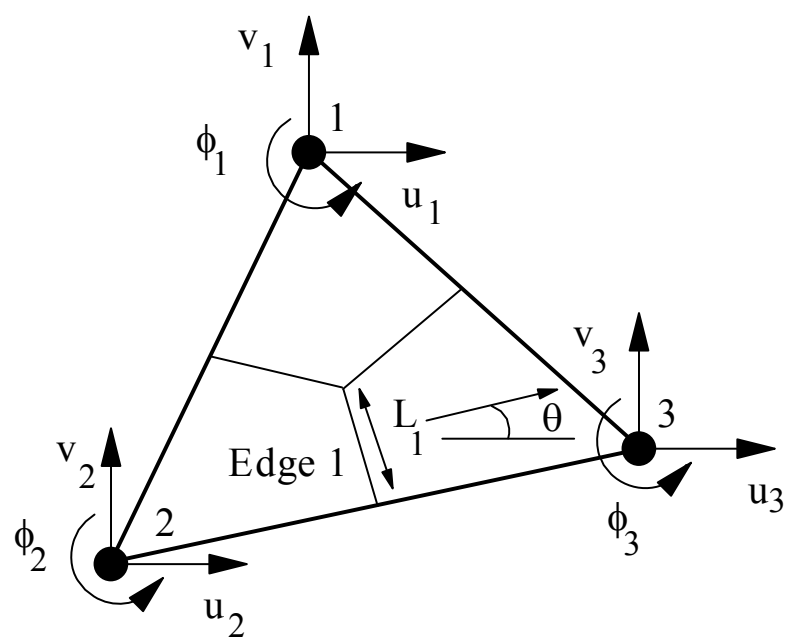

Fig 4 Triangular element with translational and rotational degrees of freedom

If the displacements and rotations are assumed vary linearly within each element then at some internal point, $\left(\xi_{1}, \xi_{2}, \xi_{3}\right)$, u, v and $\phi$ are given by

$$
\left[\begin{array}{l}
u \\
v \\
\phi
\end{array}\right]=\left[\begin{array}{lll}
u_{1} & u_{2} & u_{3} \\
v_{1} & v_{2} & v_{3} \\
\phi_{1} & \phi_{2} & \phi_{3}
\end{array}\right]\left[\begin{array}{l}
\xi_{1} \\
\xi_{2} \\
\xi_{3}
\end{array}\right]
$$

where $\xi_{1}, \xi_{2}$ and $\xi_{3}$ are the usual area or natural coordinates associated with triangular elements [25]. If these expressions are differentiated with respect to the global (x,y) coordinate system then from 14 the strains at the point $\left(\xi_{1}, \xi_{2}, \xi_{3}\right)$ are given by:- 


$$
\left[\begin{array}{c}
\varepsilon_{x} \\
\varepsilon_{y} \\
\varepsilon_{y x} \\
\varepsilon_{x y}
\end{array}\right]=\frac{1}{2 A} \mathbf{B u}+\left[\begin{array}{ccc}
0 & 0 & 0 \\
0 & 0 & 0 \\
\xi_{1} & \xi_{2} & \xi_{3} \\
-\xi_{1} & -\xi_{2} & -\xi_{3}
\end{array}\right] \phi
$$

where $A$ denotes the element area, $\mathbf{u}$ and $\phi$ are the nodal displacement $\left(\mathbf{u}=\left[\begin{array}{llllll}u_{1} & v_{1} & u_{2} & v_{2} & u_{3} & v_{3}\end{array}\right]^{T}\right)$ and rotation $\left(\phi=\left[\begin{array}{lll}\phi_{1} & \phi_{2} & \phi_{3}\end{array}\right]^{T}\right)$ vectors respectively, the matrix $\mathbf{B}$ is:-

$$
\mathbf{B}=\left[\begin{array}{cccccc}
y_{23} & 0 & y_{31} & 0 & y_{12} & 0 \\
0 & x_{32} & 0 & x_{13} & 0 & x_{21} \\
x_{32} & 0 & x_{13} & 0 & x_{21} & 0 \\
0 & y_{23} & 0 & y_{31} & 0 & y_{12}
\end{array}\right]
$$

and $y_{23}$ implies $y_{2}-y_{3}$ while $x_{32}$ means $x_{3}-x_{2}$ and so on. Equations 19 and 20 reveal that the direct strains, $\varepsilon_{x}$ and $\varepsilon_{y}$, are constant throughout an element while the shear strains, $\varepsilon_{y x}$ and $\varepsilon_{x y}$, vary linearly. Similarly, the curvatures at the point are given by:-

$$
\left[\begin{array}{c}
\phi_{, x} \\
\phi_{, y}
\end{array}\right]=\frac{1}{2 A}\left[\begin{array}{lll}
y_{23} & y_{31} & y_{12} \\
x_{32} & x_{13} & x_{21}
\end{array}\right] \phi
$$

Equations 19, 20 and 21 can then be used in conjunction with the constitutive relationships, 8 and 9 or 9 and 10, to derive expressions that describe the approximate variations in the assorted stresses throughout the element for the plane stress and plain strain cases respectively.

\subsection{Assembling the $C V$ discrete equilibrium equations}

By observing from Figure 3 that a given edge of a CV lies entirely within a particular triangular element expressions for the force and moment resultants acting on the edge can be obtained by integrating the expressions approximating the stress variations within that element along the edge. For example, performing the integrations along edge 1 of the $\mathrm{CV}$ centred on node 2 of the triangular element shown in Figure 4 yields the following expressions for the resultants:-

$$
\left[\begin{array}{l}
F_{x}^{1} \\
F_{y}^{1}
\end{array}\right]=-L_{1}\left\{\left[\begin{array}{cccc}
\cos \theta & 0 & \sin \theta & 0 \\
0 & \sin \theta & 0 & \cos \theta
\end{array}\right] \mathbf{D} \frac{1}{2 A} \mathbf{B u}+\frac{1}{6} G a\left[\begin{array}{cc}
\sin \theta & 0 \\
0 & \cos \theta
\end{array}\right]\left[\begin{array}{ccc}
2 & 5 & 5 \\
-2 & -5 & -5
\end{array}\right] \boldsymbol{\phi}\right\}
$$

and:- 


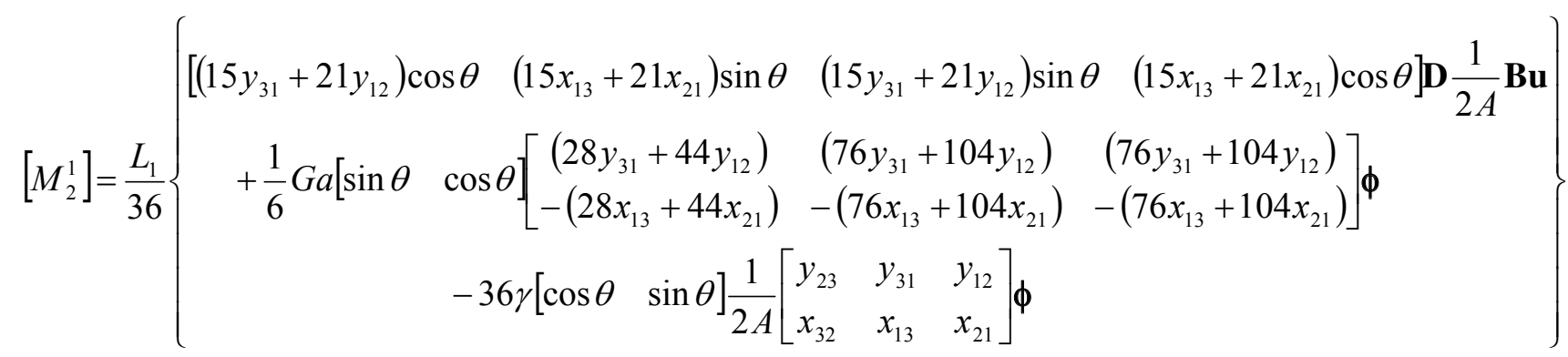

where $L_{1}$ is the length of the edge, $\theta$ is the angle that its outward normal makes with the positive $\mathrm{x}$ direction as shown in Figure 4, the matrix $\mathrm{D}$ is the 4 by 4 constitutive property array in 8 or 10 and $\gamma=2 E_{m} l^{2} /\left(1+v_{m}\right)$ from 9 . Similar expressions can be derived for the resultants acting on the second edge of this CV which, of course, is also located within the triangular element. These expressions can then be added to equations 22 and 23 to produce three equations representing the contributions that this element makes to the equilibrium equations of the $\mathrm{CV}$. The contributions that the element makes to the equilibrium equations of the CVs centred on its first and third nodes can similarly be derived. In practice these contributions are not only evaluated but are also stored on an element by element basis as in the conventional FE method. The need to obtain or store any details of the dual CV mesh is thus avoided. If the contributions that each element associated with a given $\mathrm{CV}$ are then summed as in equations 15 to 17 then the discrete equilibrium equations for that $\mathrm{CV}$ can be obtained. Obtaining these equations for all CVs is thus analogous to the assembly of a FE structural stiffness matrix from element stiffness matrices and results in a system of algebraic equations:-

$$
\left[\begin{array}{ll}
\mathbf{K}_{u u} & \mathbf{K}_{u \phi} \\
\mathbf{K}_{\phi u} & \mathbf{K}_{\phi \phi}
\end{array}\right]\left[\begin{array}{l}
\mathbf{u} \\
\boldsymbol{\phi}
\end{array}\right]=\left[\begin{array}{c}
-\mathbf{P} \\
-\mathbf{Q}
\end{array}\right]
$$

where $\mathbf{P}$ and $\mathbf{Q}$ are the body force and moment vectors respectively. Modification of equations 24 to incorporate displacement and traction boundary conditions follows conventional FE practice. An implementation of the CVFEM outlined here was developed using the MATLAB software suite and equations 24 solved using the standard algebraic equation solver supplied with the suite.

\section{Numerical Examples}

\subsection{Patch Tests}

Patch testing is employed in FE development to ensure that elements are capable of predicting simple strain states correctly. For example, a triangular element incorporating linear displacement variations and classical elastic constitutive behaviour should predict constant states of strain exactly. Patch testing thus serves as a straightforward method of checking that the results provided by an FE procedure are consistent with the assumptions made in its formulation and that its software implementation is error free. However, in the case of micropolar elasticity the notion of simple strain states is more involved. Applying a constant body moment produces linear displacement variations and a constant rotation state while applying constant 
body forces in combination with a linearly varying body moment causes displacements and rotations to vary linearly. Any FE procedure incorporating micropolar constitutive behaviour and linearly varying displacement and rotation fields should therefore pass patch tests for these states of strain. In [8] a set of three patch tests for micropolar behaviour are presented. The tests are summarized in Table 1 where the loadings applied to a rectangular membrane and the analytically obtained displacement fields are listed. The first test involves a state of constant strain in which the shear strains are complimentary, that is, $\varepsilon_{\mathrm{xy}}=\varepsilon_{\mathrm{yx}}$, while the second corresponds to a constant strain state in which they are no longer equal. The third test is more involved in that the curvatures are now constant rather than zero as they were in the previous two tests. The direct strains remain constant throughout the membrane but the shear strains now vary linearly due to the linearly varying rotation field.

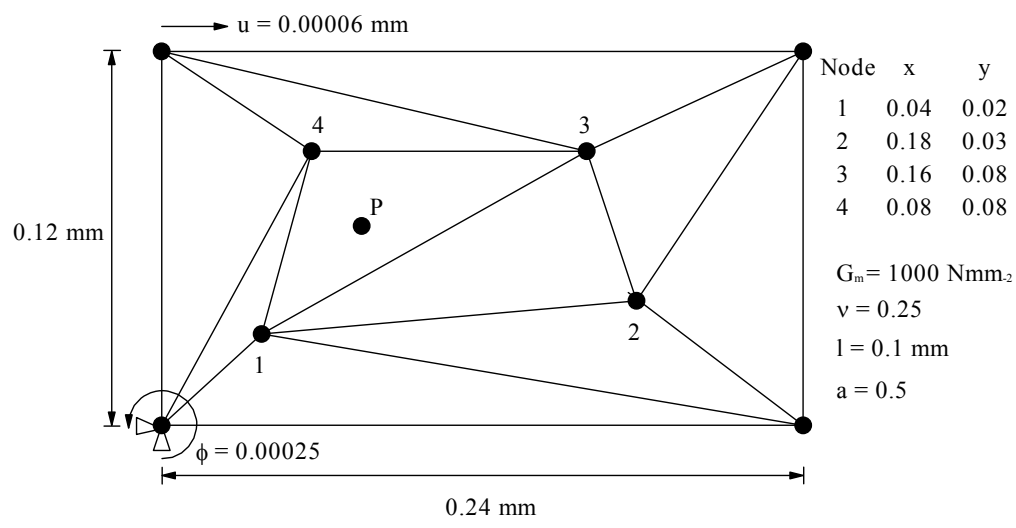

Figure 5 Mesh for Patch Tests

The elements developed in [8] were all tested using the three tests listed in Table 1 and the mesh shown in figure 5. All the elements provided exact predictions of displacements, rotations and stresses for the first two tests. However, for the third test the predictions though accurate, were acknowledged to be approximate rather than exact. The discrepancies between the numerical and analytical solutions were thought to result from the irregularity of the mesh. The three tests have also been used to verify the CVFEM presented in this paper. The mesh shown in figure 5 was used for the verifications. Displacements at the lower left node were fully fixed while the rotation at this point was prescribed according to the analytical solution listed in Table 1 as was the horizontal displacement at the upper left node. Together, these essential boundary conditions provide the minimum constraint required to obtain a unique solution for the unknowns. Nodal forces and moments were applied that were equivalent to the direct and shear stresses imposed on the periphery of the membrane and the body forces and moments acting throughout it. The CVFEM like the finite elements developed previously provides exact predictions for the first two patch tests. For the third patch test nodal displacement and rotation values and elemental stress values obtained using the CVFEM are compared to their analytically obtained counterparts in table 2. Element results presented earlier in [8] are also included in this table for comparison. The results listed clearly demonstrate that, unlike the finite elements, the CVFEM provides exact predictions of the displacement, rotation and stress fields for this test. The CVFEM is thus able to provide results that are entirely consistent with the assumed displacement and rotation variations incorporated within it. 


\subsection{Uniaxial Loading of a Plate with a Circular Hole}

The ubiquitous loaded square plate containing a central circular hole problem has also been used to assess how accurately FE methods incorporating micropolar or couple stress constitutive behaviour can predict the stress concentration around the hole $[7,8$, 9]. For a Cosserat material the problem is intriguing because the magnitude of the stress concentration depends on the degree of micropolarity; as the micropolar constitutive parameters are increased the concentration reduces [26]. In [7] this problem was analysed using triangular elements with linear displacement and rotation fields across a range of interaction intensity parameter values. For small radius holes numerical results were found to be in excellent agreement with the infinite plate analytical solution [26] across the parameter range. The size of the hole was also varied to investigate the effect of changing the radius to characteristic length ratio. Numerical results began to show some divergence from the analytical solution at larger hole sizes but this behaviour was largely attributed to the effect of the finite sized computational domain. The problem was also considered in [8] although the hole radius was fixed at a small fraction of the domain size. The performance of both linear and higher order elements was then assessed across a range of intensity parameter and characteristic length values. In general, the higher order elements provided better estimates of the stress concentration over the constitutive parameter ranges considered.

This stress concentration problem was also used to assess the performance of the CVFEM described here. Meshes with the same number of elements as those used in [8] were employed. Due to the symmetry of the problem only one quarter of the plate was considered. A typical mesh is shown in Figure 6. The element size was gradually reduced on moving in from the plate edge towards the hole. On the planes of symmetry the normal displacements and rotations were suppressed while on one of the edges nodal loads and moments consistent with a uniform normal stress were applied.

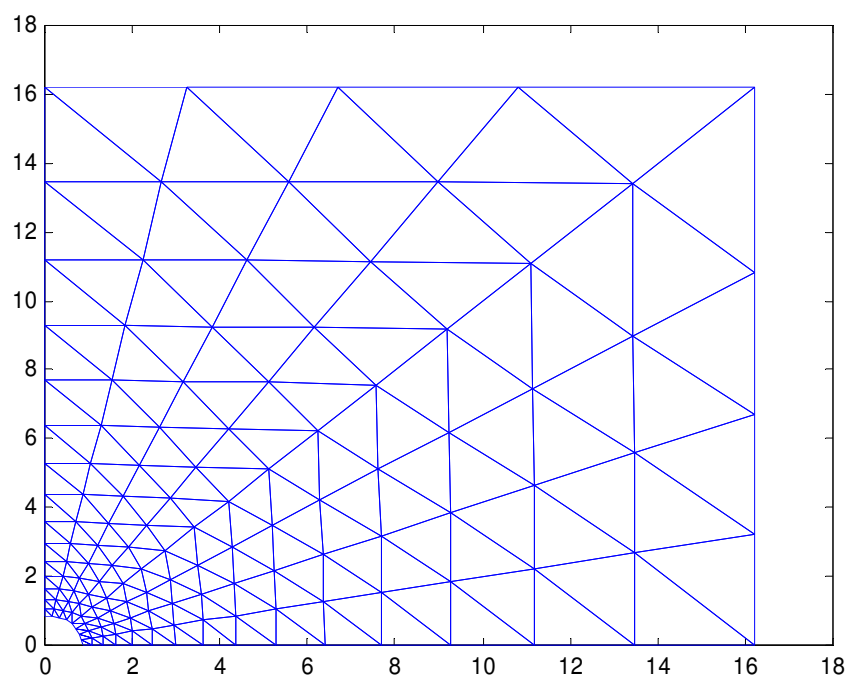


Figure 6 Mesh $(8 \times 15 \times 4)$ used to represent plate with $0.864 \mathrm{~mm}$ radius hole (quarter plate measures $16.4 \mathrm{~mm}$ by $16.4 \mathrm{~mm}$ )

For a range of values of the interaction parameter, $a$, the maximum circumferential stress in an element adjacent to the hole was determined for two different values of the hole radius to characteristic length ratio. The results are tabulated in Tables 3 and 4 where previous FE results and the analytical values for an infinite plate are also quoted. The percentage errors in the numerical solutions are listed in parentheses. From the tables it can be seen that for classical elastic behaviour $(a=0)$ the CVFEM predicts the stress concentration more accurately than the triangular element incorporating linear displacement and rotation fields (MLINT) though not as accurately as the higher order triangular elements (MQLT with quadratic displacement and linear rotation fields and MQUAT with quadratic displacement and rotation fields). As $a$ increases the accuracy of the CVFEM, like the finite elements, first improves and then, at a high value of $a$, reduces again. Table 5 compares the CVFEM and FE results for the case where $a$ is held constant while the ratio of the hole radius to characteristic length is varied by altering $l$ while fixing $r$. Here the CVFEM provides predictions that are more accurate than any of the FE methods and accuracy improves as the characteristic length is increased.

Overall, the predictions of the CVFEM are impressive in that they are always more accurate than the linear finite elements and are actually of comparable accuracy to the higher order elements across the parameter ranges. Indeed, the CVFEM might actually be more accurate than the higher order elements because the meshes used by both methods contained the same number of elements so the FE results were obtained using a larger number of degrees of freedom. However, a full convergence study of both methods would be required to confirm this.

\section{Conclusions}

A CVFEM has been successfully developed for predicting planar deformations and stresses within loaded materials exhibiting micropolar or Cosserat constitutive behaviour. The performance of the method has been assessed using patch tests and a two dimensional plane strain stress concentration problem. The patch testing has demonstrated that the method is able to predict simple strain states exactly and therefore the results it provides are consistent with the assumptions incorporated in it. For the stress concentration problem the accuracy to which the method predicts the magnitude of the concentration was determined across a range of micropolar constitutive parameters. A comparison of the predictions provided by the method with those given previously by FE methods has revealed that its accuracy is superior to the equivalent low order FE method and is actually comparable to higher order methods. As mentioned earlier, enhancing existing FE methods to analyze more challenging three dimensional situations has still to be realized. The results reported here support the enhancement of control volume based methods as an alternative in meeting this challenge and research is currently underway to address this.

\section{References}

[1] E.C. Aifantis, On the microstructural origin of certain inelastic models, ASME J. Eng. Mat. Tech., 106 (1984), 326-330 
[2] A.C. Eringen, Linear theory of micropolar elasticity, Int. J. Math. Mech., 15 (1966), 909-923

[3] J.Y. Shu, W.E. King \& N.A. Fleck, Finite elements for materials with strain gradient effects, Int. J. Num. Meth. Eng., 44 (1999), 373-391

[4] A. Zervos, P. Papanastasiou \& I. Vardoulakis, A finite element displacement formulation for gradient elastoplasticity, Int. J. Num. Meth. Eng., 50 (2001), 13691388

[5] A.K. Soh \& C. Wanji, Finite element formulations of strain gradient theory of microstructures and the $\mathrm{C}^{0-1}$ patch test, Int. J. Num. Meth. Eng., 61 (2004), 433-454

[6] P. Steinmann, An improved FE expansion for microploar localization, Comm. Num. Meth. Eng., 10 (1994), 1005-1012

[7] S. Nakamura, R. Benedict \& R. Lakes, Finite element method for orthotropic micropolar elasticity, Int. J. Eng. Sci., 22 (1984), 319-330

[8] E. Providas \& M.A. Kattis, Finite element method in plane Cosserat elasticity, Comput. Struct., 80 (2002), 2059-2069

[9] L. Li \& S. Xie, Finite element method for linear micropolar elasticity and numerical study of some scale effects phenomena in MEMS, Int. J. Mech. Sci., 46 (2004), 1571-1587

[10] E. Diegele, R. Elsässer \& C. Tsakmakis, Linear micropolar elastic crack tip fields under mixed mode loading conditions, Int. J. of Fracture 129 (2004) 309-339

[11] B.R. Baliga \& S.V. Patankar, A new finite element formulation for convection diffusion problems, Numerical Heat Transfer, 3 (1980), 393-409

[12] S.V. Patankar, Numerical Fluid Flow and Heat Transfer, Hemisphere, Washington D.C., 1980

[13] A.G. Malan \& R.W. Lewis, Modelling coupled heat and mass transfer in drying non hygroscopic capillary particulate materials, Comm. Num. Meth. Eng., 19 (2003), 669-677

[14] C. Bailey \& M. Cross, A finite volume procedure to solve elastic solid mechanics problems in three dimensions on an unstructured mesh, Int. J. Num. Meth. Eng., 38 (1995), 1757-1776

[15] C.J. Greenshields, G.P. Venizelos \& A. Ivankovic, A fluid structure model for fast brittle fracture in plastic pipes, J. Fluids \& Struct., 14 (2000), 221-234

[16] C. J. Greenshields \& H. G. Weller, A unified formulation for continuum mechanics applied to fluid-structure interaction in flexible tubes, Int. J. Num. Meth. Eng., 64 (2005), 1575-1593

[17] A.K. Slone, K. Pericleous, C. Bailey \& M. Cross, Dynamic fluid-structure interaction using finite volume unstructured mesh procedures, Comput. Struct., 80 (2002), 371-390

[18] A. Ivankovic, I. Demirdzic, J.G. Williams and P.S. Leevers, Application of the finite volume method to the analysis of dynamic fracture problems, Int. J. of Fracture 66 (1994) 357-371.

[19] G.A. Taylor, C. Bailey, C. and M. Cross, 'Solution of the elastic visco plastic constitutive equations: a finite volume approach', Appl. Math. Modelling 19 (1995), 747-760, 1995

[20] N.A. Fallah, C. Bailey, M. Cross \& G.A. Taylor, Comparison of finite element and finite volume methods application in geometrically nonlinear stress analysis, Appl. Math. Modelling 24 (2000), 439-455

[21] I. Bijelonja, I. Demirdzic \& S. Muzaferija, A finite volume method for large strain analysis of incompressible hyperelastic materials, Int. J. Num. Meth. Eng., 64 (2005), 1594-1609 
[22] P. Wenke \& M.A. Wheel, A finite volume method for solid mechanics incorporating rotational degrees of freedom, Comput. Struct., 81 (2003), 321-329

[23] D.J. Allman, A compatible triangular element including vertex rotations for plane elasticity analysis, Comput. Struct., 19 (1984), 1-8

[24] S. Nakamura, \& R.S. Lakes, Finite element analysis of Saint Venant end effects in micropolar elastic solids, Eng. Computat., 12 (1995), 571-587

[25] R.D. Cook, D.S. Malkus \& M.E. Plesha, Concepts and applications of finite element analysis, Third edition, Chapter 5, John Wiley, New York, 1989

[26] P.N. Kaloni \& T. Ariman, Stress concentration effects in micropolar elasticity, ZAMP, 18 (1967), 136-141

\begin{tabular}{|l|}
\hline Test 1:- \\
Loading:- $\mathrm{p}_{\mathrm{x}}=\mathrm{p}_{\mathrm{y}}=\mathrm{q}=0, \sigma_{\mathrm{x}}=\sigma_{\mathrm{y}}=4, \tau_{\mathrm{xy}}=\tau_{\mathrm{yx}}=1.5, \mathrm{~m}_{\mathrm{x}}=\mathrm{m}_{\mathrm{y}}=0$ \\
Solution:- $\mathrm{u}=10^{-3}\{\mathrm{x}+(1 / 2) \mathrm{y}\}, \mathrm{v}=10^{-3}\{\mathrm{x}+\mathrm{y}\}, \phi=(1 / 4) \times 10^{-3}$ \\
\hline Test 2:- \\
Loading:- $\mathrm{p}_{\mathrm{x}}=\mathrm{p}_{\mathrm{y}}=0, \mathrm{q}=1, \sigma_{\mathrm{x}}=\sigma_{\mathrm{y}}=4, \tau_{\mathrm{xy}}=1, \tau_{\mathrm{yx}}=2, \mathrm{~m}_{\mathrm{x}}=\mathrm{m}_{\mathrm{y}}=0$ \\
Solution:- $\mathrm{u}=10^{-3}\{\mathrm{x}+(1 / 2) \mathrm{y}\}, \mathrm{v}=10^{-3}\{\mathrm{x}+\mathrm{y}\}, \phi=10^{-3}\{(1 / 4)+(1 / 4 \alpha)\}, \alpha=0.5$ \\
\hline Test 3:- \\
Loading:- $\mathrm{p}_{\mathrm{x}}=\mathrm{p}_{\mathrm{y}}=1, \mathrm{q}=2(\mathrm{x}-\mathrm{y}), \sigma_{\mathrm{x}}=\sigma_{\mathrm{y}}=4, \tau_{\mathrm{xy}}=1.5-(\mathrm{x}-\mathrm{y}), \tau_{\mathrm{yx}}=1.5+(\mathrm{x}-\mathrm{y})$, \\
$\mathrm{m}_{\mathrm{x}}=-\mathrm{m}_{\mathrm{y}}=\left(2 l^{2} / \alpha\right), \alpha=0.5$ \\
Solution:- $\mathrm{u}=10^{-3}\{\mathrm{x}+(1 / 2) \mathrm{y}\}, \mathrm{v}=10^{-3}\{\mathrm{x}+\mathrm{y}\}, \phi=10^{-3}\{(1 / 4)+(1 / 2 \alpha)(\mathrm{x}-\mathrm{y})\}$ \\
\hline
\end{tabular}

Table 1 Patch tests for micropolar materials:- body and boundary loadings and polynomial solutions for displacement and rotation fields

\begin{tabular}{|c|c|c|c|c|c|c|}
\hline Method & $\mathrm{u}\left(\times 10^{3}\right)$ & $\mathrm{v}\left(\times 10^{3}\right)$ & $\phi\left(\times 10^{3}\right)$ & $\sigma_{\mathrm{x}}$ & $\sigma_{\mathrm{y}}$ & $\mathrm{m}_{\mathrm{x}}$ \\
\hline MLINT & 0.1944 & 0.2096 & 0.4001 & 3.9928 & 1.4634 & 0.0400 \\
\hline MQLT & 0.1945 & 0.2097 & 0.4001 & 3.9956 & 1.4668 & 0.0400 \\
\hline MQUAT & 0.1945 & 0.2097 & 0.3960 & 3.9956 & 1.4707 & 0.0392 \\
\hline CVFEM & 0.195000 & 0.210000 & 0.400000 & 4.000000 & 1.466666 & 0.040000 \\
\hline Exact & 0.195000 & 0.210000 & 0.400000 & 4.000000 & 1.466666 & 0.040000 \\
\hline
\end{tabular}

Table 2 Patch test 3:- Comparison of displacements at node 2 and stresses at point $P$ predicted by FE methods [7] and CVFEM with exact solutions.

\begin{tabular}{|c|c|c|c|c|c|}
\hline$a$ & Analytical & $\begin{array}{c}\text { FE } \\
\text { (MLINT) }\end{array}$ & FE (MQLT) & $\begin{array}{c}\text { FE } \\
\text { (MQUAT) }\end{array}$ & CVFEM \\
\hline 0.0 & 3.000 & $2.867(4.4)$ & $3.027(0.9)$ & $3.027(0.9)$ & $2.904(3.2)$ \\
\hline 0.0667 & 2.849 & $2.753(3.4)$ & $2.874(0.9)$ & $2.875(0.9)$ & $2.783(2.3)$ \\
\hline 0.3333 & 2.555 & $2.516(1.5)$ & $2.576(0.8)$ & $2.577(0.9)$ & $2.532(0.9)$ \\
\hline 1.2857 & 2.287 & $2.269(0.8)$ & $2.303(0.7)$ & $2.306(0.9)$ & $2.279(0.3)$ \\
\hline 4.2632 & 2.158 & $2.103(2.6)$ & $2.174(0.8)$ & $2.179(1.0)$ & $2.124(1.6)$ \\
\hline
\end{tabular}

Table 3 Maximum Circumferential Stress at Circular Hole Predicted by CVFEM and various finite elements [7] (hole radius $0.216 \mathrm{~mm}, r / l=1.063, v=0.3$, mesh $8 \times 22 \times 4$ ) 


\begin{tabular}{|c|c|c|c|c|c|}
\hline$a$ & Analytical & $\begin{array}{c}\text { FE } \\
\text { (MLINT) }\end{array}$ & FE (MQLT) & $\begin{array}{c}\text { FE } \\
\text { (MQUAT) }\end{array}$ & CVFEM \\
\hline 0.0 & 3.000 & $2.867(4.4)$ & $3.027(0.9)$ & $3.027(0.9)$ & $2.904(3.2)$ \\
\hline 0.0667 & 2.956 & $2.833(4.2)$ & $2.987(0.8)$ & $2.983(0.9)$ & $2.922(1.1)$ \\
\hline 0.3333 & 2.935 & $2.809(4.3)$ & $2.969(1.2)$ & $2.966(0.9)$ & $2.932(0.1)$ \\
\hline 1.2857 & 2.927 & $2.761(5.7)$ & $2.983(1.9)$ & $2.973(1.6)$ & $2.924(0.1)$ \\
\hline 4.2632 & 2.923 & $2.633(9.9)$ & $2.975(1.8)$ & $3.005(2.8)$ & $2.846(2.6)$ \\
\hline
\end{tabular}

Table 4 Maximum Circumferential Stress at Circular Hole Predicted by CVFEM and various finite elements [7] (hole radius $0.216 \mathrm{~mm}, r / l=10.63, v=0.3$, mesh $8 \times 22 \times 4$ )

\begin{tabular}{|c|c|c|c|c|c|}
\hline$r / l$ & Analytical & $\begin{array}{c}\text { FE } \\
(\text { MLINT) }\end{array}$ & FE (MQLT) & $\begin{array}{c}\text { FE } \\
(\text { MQUAT) }\end{array}$ & CVFEM \\
\hline 10.0 & 2.929 & $2.824(3.6)$ & $2.984(1.9)$ & $2.981(1.8)$ & $2.957(1.0)$ \\
\hline 8.0 & 2.902 & $2.804(3.4)$ & $2.954(1.8)$ & $2.952(1.7)$ & $2.922(0.7)$ \\
\hline 6.0 & 2.857 & $2.770(3.1)$ & $2.905(1.7)$ & $2.905(1.7)$ & $2.868(0.4)$ \\
\hline 4.0 & 2.779 & $2.709(2.5)$ & $2.821(1.5)$ & $2.823(1.6)$ & $2.781(0.1)$ \\
\hline 3.0 & 2.719 & $2.661(2.1)$ & $2.758(1.5)$ & $2.761(1.6)$ & $2.717(0.1)$ \\
\hline 2.0 & 2.641 & $2.599(1.6)$ & $2.678(1.4)$ & $2.680(1.5)$ & $2.638(0.1)$ \\
\hline 1.0 & 2.549 & $2.524(1.0)$ & $2.584(1.4)$ & $2.585(1.4)$ & $2.547(0.1)$ \\
\hline
\end{tabular}

Table 5 Maximum Circumferential Stress at Circular Hole Predicted by CVFEM and various finite elements [7] (hole radius $0.216 \mathrm{~mm}, a=1 / 3, v=0.3$, mesh $8 \times 15 \times 4$ ) 